\title{
Protocol for a Systematic Review of Outcomes \\ From Microsurgical Free Tissue Transfer Performed on Short-term Surgical Missions in Low-income and Middle-income Countries
}

\author{
Henry Tobias de Berker ( $\sim$ h.deberker@doctors.org.uk) \\ Wythenshawe Hospital https://orcid.org/0000-0002-8307-0818 \\ Urška Čebron
}

Department of Hand, Plastic and Reconstructive Surgery, University of Tubingen

\section{Daniel Bradley}

King's College London, Faculty of Dentistry, Oral and Craniofacial Sciences, Guy's Tower, Guy's Hospital, London

\section{Vinod Patel}

Oral Surgery Department, Guy's Dental Institute, Guy's and St Thomas NHS Foundation Trust, London

\section{Meklit Berhane}

Department of Plastic and Reconstructive Surgery, ALERT Hospital, Addis Ababa

\section{Fernando Almas}

Department of Cranio-Maxillofacial and Reconstructive Surgery, Saint Jude's General Hospital, Federal Hospital of Rio de Janeiro, Rio de Janeiro

\section{Gary Walton}

Department of Head and Neck Surgery, University Hospitals Coventry and Warwickshire

\section{Mekonen Eshete}

Department of Surgery, School of Medicine, College of Health Sciences, Addis Ababa University

\section{Mark McGurk}

Deparment of Head and Neck Surgery, University College London Hospital, London

\section{Dominique Martin}

Private practice, 35 Avenue Des Pins 13013, Marseille

\section{Calum Honeyman}

Department of Plastic, Reconstructive and Burns Surgery, Ninewells Hospital, Dundee

\section{Protocol}

Keywords: Free flap, Free tissue transfer, Microsurgery, Short-term surgical missions, Humanitarian missions, Low-income and middle-income countries, Resource limited settings 
Posted Date: January 15th, 2021

DOI: https://doi.org/10.21203/rs.3.rs-144661/v1

License: (c) (i) This work is licensed under a Creative Commons Attribution 4.0 International License. Read Full License 


\section{Abstract}

Background: In many units around the world, microsurgical free tissue transfer represents the gold standard for reconstruction of significant soft tissue defects following cancer, trauma or infection. However, many reconstructive units in low-income and middle-income countries (LMICs) do not yet have access to the resources, infrastructure or training required to perform any microsurgical procedures. Longterm international collaborations have been formed with annual short-term reconstructive missions conducting microsurgery. In the first instance, these provide reconstructive surgery to those who need it. In the longer-term, they offer an opportunity for teaching and the development of sustainable local services.

Methods: A PRISMA-compliant systematic review and meta-analysis will be performed. A comprehensive, predetermined search strategy will be applied to the MEDLINE and Embase electronic databases from inception to December 2020. All clinical studies presenting sufficient data on free tissue transfer performed on short-term surgical missions (STSMs) in LMICs will be eligible for inclusion. The primary outcomes are rate of free flap failure, rate of emergency return to theatre for free flap salvage and successful salvage rate. The secondary outcomes include postoperative complications and any functional or patient reported outcome measures. Screening of studies, data extraction and assessments of study quality and bias will be conducted by two authors. Individual study quality will be assessed according to the Oxford Evidence-based Medicine Scales of Evidence 2, and risk of bias using either the 'Revised Cochrane risk of bias tool for randomized trials' (Rob2), or the 'Risk of bias in non-randomized studies of interventions' (ROBINS-I) tools. Overall strength of evidence will be assessed according to the Grading of Recommendations, Assessment, Development and Evaluations (GRADE) approach.

Discussion: To-date the outcomes of microsurgical procedures performed on STSMs to LMICs are largely unknown. Improved education, funding and allocation of resources are needed to support surgeons in LMICs to perform free tissue transfer. STSMs provide a vehicle for sustainable collaboration and training. Disseminating microsurgical skills could improve the care received by patients living with reconstructive pathology in LMICs, but this is poorly established. This study sets out a robust protocol for a systematic review designed to critically analyse outcomes.

Systematic review registration: Submitted to PROSPERO (15/12/20 ID: 225613)

\section{Background}

Five billion people around the world are living without access to adequate surgical care(1). Short-term surgical missions (STSMs) provide an opportunity for surgical teams from higher-income nations to collaborate with surgeons from low-income and middle-income countries (LMICs) to treat those most in need. Historically, some STSMs have been criticised for an unsustainable 'fly-in-fly-out' model of surgical care delivery in LMICs, with limited patient follow-up after discharge. One consequence of this approach is a poor evidence base by surgeons working in resource-limited settings are able to decide on the best 
treatment options for their patients.(2). However, most STSMs return annually to the same centre, building lasting relationships, offering sustainable education and training, and treating patients who would otherwise have no access to healthcare. This model offers greater opportunity for thorough followup. Indeed, new methods of long-term patient follow-up after discharge are emerging that aim to improve patient safety after STSMs in LMICs(3, 4).

Free-tissue transfer can be considered the gold-standard method of reconstruction after significant composite tissue defects from cancer, trauma and infections(5-8). In the largest series from high volume centres, flap failure rates as low as $0.6 \%$, and take back rates of of $1.5 \%$ ( $66 \%$ successful salvage rate) have been published(9). For surgeons operating in LMICs, that do not have funding and access to the required equipment and training to facilitate independent microsurgical practice, treatment options are restricted to those used 50 years ago in countries that now perform regular free tissue transfer. For select advanced pathology, this may be the difference between amputation and lower limb salvage after trauma and sarcoma resection, or poorer functional outcomes following head and neck cancer reconstruction(10-14). Performing microsurgery in LMICs is challenging, even for experienced teams. However, a select few centres in LMICs are undertaking these procedures as part of standard practice(15-17). Local and regional alternatives do exist, however many of these are also technically demanding, and even with extensive training, microsurgical equipment and techniques, they are vulnerable to complications requiring further surgery $(18-21)$. However, there is also some data in support of successful local reconstructive alternatives being used effectively in low-resource contexts(21).

There are some fundamental challenges to providing microsurgery in LMICs. Amongst those reported are a lack of specialist equipment, trained staff and appropriate level three post-operative monitoring facilities(22). STSMs, which can provide additional staff and resources, can directly address these challenges. However, these are usually short-term interventions, and patients may not be afforded the same degree of follow-up provided by the home institutions of the visiting surgeons. In addition, it is traditionally held that reconstructive surgeons should stick to 'simple' surgery on STSMs(23). Therefore, it is important to establish the safety of delivering free tissue transfer on STSMs. Identification of common complications and constraints will inform organisations engaged in STSMs where to focus their training and fundraising efforts in order to improve patient outcomes.

The aim of this study is to publish a robust systematic review protocol to establish the safety and efficacy of microsurgical free tissue transfer performed on STSMs in low-income and middle-income countries.A meta-analysis of key outcomes will be undertaken with the aim of developing potentially life changing microsurgical practice in resource-limited settings.

\section{Methods}

This protocol is registered on the PROSPERO international prospective register of systematic reviews (Submitted 15/12/20 ID: 225613) and is written in accordance with the Preferred Reporting Items for Systematic Reviews and Meta-Analysis Protocols (PRISMA-P) guidelines $(24,25)$. The methodology 
applied to the final systematic review and meta-analysis is derived from, and in line with, the Cochrane Handbook for Systematic Review of Interventions(26) and the Preferred Reporting Items for Systematic Reviews and Meta-Analyses (PRISMA) statement (27).

\section{Search strategy}

The search strategy (Table 1) will be applied to the MEDLINE and Ovid EMBASE databases from inception to $2^{\text {nd }}$ January 2021.

Table 1.

Electronic database search - MEDLINE and Ovid EMBASE

\begin{tabular}{|c|c|c|c|c|c|}
\hline & Concept 1 - Surgery & AND & Concept 2 - Micro & AND & Concept 3 - STSM \\
\hline MeSH term & Surgery & & Free Tissue Flaps & & Medical Missions \\
\hline \multirow[t]{12}{*}{ OR } & & & Microsurgery & & \\
\hline & & OR & Flap* & OR & Mission* \\
\hline & & OR & Free Flap* & OR & Humanitarian \\
\hline & & OR & Free Tissue Transfer & OR & Visit* \\
\hline & & & & OR & Charit* \\
\hline & & & & OR & Trip \\
\hline & & & & OR & Outreach \\
\hline & & & & OR & Volunt* \\
\hline & & & & OR & Non-government* \\
\hline & & & & OR & Safari \\
\hline & & & & OR & Blitz \\
\hline & & & & OR & Camp \\
\hline
\end{tabular}

\section{Study selection criteria}

\section{Inclusion criteria}

All clinical studies reporting outcomes of any microsurgical procedure performed on a short-term reconstructive mission to any low-income or middle-income country (in accordance with the world bank classification(28)) will be eligible for inclusion. Studies that match the inclusion criteria performed in lowresource environments in a high-income country will also be included. Children and adults will be considered. All cases performed using either operating microscope or loupe magnification will be 
included, as both are successfully reported in resource-limited settings(15). The Population, Intervention, Comparison, Outcome (PICO) model was used to determine study selection criteria (Table 2)(29).

Table 2.

Population, Intervention, Comparison, Outcome (PICO)

\begin{tabular}{|llll|}
\hline Population & Intervention & Comparator & Outcomes \\
\hline $\begin{array}{l}\text { Patients operated on during } \\
\text { STSMs }\end{array}$ & $\begin{array}{l}\text { Reconstructive surgery by } \\
\text { microvascular free tissue } \\
\text { transfer }\end{array}$ & $\begin{array}{l}\text { Data from the UK } \\
\text { National Flap } \\
\text { Registry(8) }\end{array}$ & $\begin{array}{l}\text { Rate of } \\
\text { complications }\end{array}$ \\
\hline $\begin{array}{l}\text { In low- and middle-income } \\
\text { countries or low-resource health } \\
\text { environments }\end{array}$ & $\begin{array}{l}\text { Performed during a } \\
\text { visiting surgical mission }\end{array}$ & & $\begin{array}{l}\text { Nature of } \\
\text { complications }\end{array}$ \\
$\begin{array}{l}\text { Children and adults to be } \\
\text { included }\end{array}$ & $\begin{array}{l}\text { This will include both } \\
\text { loupes and microscope } \\
\text { magnification }\end{array}$ & $\begin{array}{l}\text { Rate of return } \\
\text { to theatre }\end{array}$ \\
\hline & Rate of free \\
& & & $\begin{array}{l}\text { Rap salvage } \\
\text { flap }\end{array}$ \\
\hline
\end{tabular}

\section{Exclusion criteria}

Studies that do not provide sufficient data for comparative analysis will be excluded. Where incomplete or absent data is presented in a given study, study authors will be contacted by email on a maximum of two separate occasions, two weeks apart, inviting them to provide further data before being excluded. Data presented from microsurgical units already independently performing microsurgery in low-income or middle-income countries, not on a STSM, will also be excluded.

\section{Study design}

Randomised controlled trials (RCTs), cohort, case-control and case series will be considered for inclusion. Pilot searches indicate that the majority of studies will be case series of varying sizes. As such, no limitation regarding study size or clinical follow-up will be made. Case reports, letters, opinion pieces and literature reviews will be excluded. Any unpublished or ongoing prospective clinical trials will also be excluded.

\section{Participants}

Children or adults that have undergone microsurgical free tissue transfer during a short-term surgical mission in a low-income and middle-income country. No limitations based on patient demographics, body 
region or aetiology will be imposed.

\section{Outcomes}

\section{Primary outcomes}

The primary outcomes are rate of free flap failure and rate of emergency return to theatre for attempted free flap salvage. Return to theatre will be classified as a donor site or anastomotic complication. The time taken to return to theatre, and the rate of successful flap salvage will also be documented where available. Where disclosed, we will record the method of flap salvage attempted.

\section{Secondary outcomes}

The secondary outcomes will include complications and any functional or patient reported outcome measures included. Based on previous large case studies of free flaps, complications will be divided into medical and surgical. The surgical group will be further subdivided into intraoperative and post-operative. Complications will be stratified according to the Clavien-Dindo classification(30-32). Finally, if available any assessment of pre-operative fitness will be recorded.

\section{Additional data}

In addition to the primary and secondary outcomes, duration of procedure and length of stay will also be recorded where available. In the context of a STSM to a LMIC, this is particularly important. The following will also be extracted: bibliographic data (Title, author, date), study characteristics (design, method of randomisation/allocation, blinding, number of participants, groups/subgroups), mission characteristics (country, length of mission, type of mission/subspecialty, organisation (type and size), type of hospital (e.g. public or private), frequency of missions), patient characteristics (age, sex, indication for surgery, comorbidities, smoking status), intervention characteristics (Operation(s) performed, duration of operation, length of stay, who performed the surgery (local or visiting surgeon), experience level of surgeon who performed anastomosis and raised flap, pre-operative workup), and rate and duration of follow up.

Outcomes will be compared to data available from the multicentre, UK National Flap Registry, published in 2019(8).

\section{Data management and extraction}

Abstracts will be screened on the Rayyan systematic review software tool. Full papers will be downloaded as PDFs, and stored locally on Mendeley Desktop. All abstracts included will proceed to full text analysis unless it is immediately apparent following reading of the introduction that they are irrelevant. Data items will be collected in a standardised data collection proforma. For instances of incomplete data, we will contact the corresponding author. If 2 weeks elapse with no response, we will repeat this request once.

\section{Data selection}


Screening will be conducted and recorded in accordance with the Preferred Reporting Items for Systematic Review and Meta-Analysis (PRISMA) guidelines(27). Abstracts screened according to criteria set out in this protocol by two independent researchers ( $\mathrm{HdB}$ and $\mathrm{UC}$ ). In case of disagreements: researchers will meet to discuss disparities; if there are still disagreements, a third author $(\mathrm{CH})$ will make the final decision on inclusion. If abstracts are not available on assessment, the paper will be downloaded in full for analysis.

\section{Risk of bias assessment}

Each study will be assessed for risk of bias by two independent reviewers (HdB and UC) according to an appropriate validated tool. Randomised studies will be assessed using the revised Cochrane risk of bias tool for randomized trials (Rob2)(33). Non-randomised studies will be assessed using the 'Risk of bias in non-randomized studies of interventions' (ROBINS-I) tool(34).

\section{Quality of studies}

Each study will be assessed according to the Oxford Evidence-based Medicine Scales of Evidence 2(35). This data will be tabulated.

\section{Strategy for data analysis and synthesis}

Statistical analysis of included studies will be undertaken in Revman 5.4 (Cochrane Collaboration, Oxford, United Kingdom). Patient demographics will be presented using basic descriptive statistics. Complications, free flap failure, emergency return to theatre and successful free flap salvage will be calculated and displayed as rate (\%). Data from the first UK National flap registry will be used as a comparator(8). Statistical heterogeneity will be examined by calculating ${ }^{2}$ and Cochran's $Q$ statistic, and interpreted according to Cochrane guidance on determining heterogeneity(26). If $\mathrm{I}^{2}>50$, a random-effects model (DerSimonian and Laird with a logit transformation applied) will be used to calculate relative risk with $95 \%$ confidence intervals(36). If $\mathrm{I}^{2} \leq 50$, a fixed-effects model will be used for relative risk calculations. A $p$-value of $<0.05$ will be considered statistically significant. The results of this metaanalysis will be presented in Forest plots. Funnel plots will be used to detect publication bias. If quantitative analysis is inappropriate a narrative synthesis will be performed.

The analysis detailed above will be undertaken for the pooled data. If sufficient data is available, subgroup analysis by region (e.g. head and neck, trunk etc.) will be undertaken.

\section{Confidence in cumulative evidence}

The body of evidence underpinning each of the findings will be assessed according to the 'Grading of Recommendations, Assessment, Development and Evaluations' (GRADE) approach(37). Using this approach, the authors will express their 'certainty' that the body of evidence reflects reality (High, moderate, low, very low). 


\section{Discussion}

This will be the first systematic review and meta-analysis of the outcomes of free tissue transfer performed on STSMs to low-income and middle-income countries. Our pilot searches indicate that the majority of data will be published in case series', it is difficult to form a conclusion from any one of these alone. This underlines the importance of a thorough synthesis of these studies into a unifying review article. However, lack of randomised allocation and control groups increase the risk of bias, and this has led to concerns regarding the quality of evidence in this field(38). We cannot remedy this, but will provide the reader with an open appraisal of the body of evidence according to the GRADE approach. From this they will be able to form their own conclusions about their confidence in our findings.

We predict considerable clinical heterogeneity between the studies included in this paper. STSMs are often based on relationships between specific institutions, sometimes individual surgeons, and thus there is no 'one size fits all approach'. Another source of heterogeneity will come from the inclusion of all flap types. We will try to mitigate this through sub-group analysis if possible, but there may be insufficient data available.

Nutritional status has been identified as a determinant of free flap outcome(39). STSMs treat a diverse population of patients, many of whom will have poor nutritional status. This may be a confounding factor in reconstructive outcomes, but is unlikely to be well documented in a standardised manner across the literature. We will collect nutritional and other preoperative assessments where possible, but there may not be sufficient data to adjust for these variables.

We hope to equip surgeons undertaking STSMs with the relevant data to offer appropriate treatment to their patients in order to achieve the best possible outcomes. In addition, this will ensure patients are able to give informed consent with an understanding of the risks specific to their situation. These impacts will improve patient safety, reconstructive outcomes and follow-up.

Finally, with appropriately strict governance surrounding distribution of funds designed to provide healthcare in low- and middle-income countries, it is essential that actors in this field are able to provide evidence supporting their work. Through identifying the nature and severity of complications, we hope to inform surgeons and funders of the challenges and barriers to free tissue transfer in LMICs. This should encourage investment in areas that are most likely to improve patient outcomes. This study will assist those funding global surgery to allocate resources to appropriate interventions with proven patient benefit.

\section{Abbreviations}

EMBASE Elsevier Biomedical and Pharmacological Bibliographic Database

GRADE Grading of Recommendations, Assessment, Development and Evaluations 
LMICs Low- and Middle-Income Countries

MEDLINE US National Library of Medicine Bibliographic Database

MeSH Medical Subject Headings

PRISMA Preferred Reporting Items for Systematic Reviews and Meta-Analyses

PROSPERO International prospective register of systematic reviews

RCT Randomised controlled trial

Rob2 Revised Cochrane risk of bias tool for randomized trials

Robins-I Risk of bias in non-randomized studies of interventions

STSM Short-Term Surgical Mission

\section{Declarations}

\section{Acknowledgements}

The authors would like to express our warmest gratitude to the staff and volunteers working for Project Harar in Ethiopia and the United Kingdom. We would also like to express our thanks to the plastic surgery team at Yekatit-12 Hospital and Medical College, Addis Ababa for their ongoing support and engagement.

\section{Availability of data and materials}

Data sharing is not applicable to this article as no datasets were generated or analysed during the current study.

\section{Ethics approval and consent to participate}

Not applicable

\section{Consent for publication}

Not applicable

\section{Availability of data and materials}

Data sharing is not applicable to this article as no datasets were generated or analysed during the current study.

\section{Competing interests}


All of the authors have volunteered for the non-governmental organisation Project Harar, which conducts STSMs. VP and MM are trustees for Project Harar, and both sit on the board of directors

\section{Funding}

There are no sources of additional funding for this research

\section{Authors' contributions}

All of the listed authors have made a substantial contribution toward the development of this protocol. $\mathrm{CH}$ conceived the study, reviewed the protocol design and registration, took part in the manuscript drafting and completion and gave specialist input relating to microsurgery and STSMs in LMICs. HdB developed the search strategy and data analysis section, reviewed the protocol design and registration, took part in the manuscript drafting and completion. DB,VP, MB, FA, GW, MM, DM, ME and VP provided expertise in the methodology and clinical area covered. $\mathrm{HdB}, \mathrm{UC}$ developed the search strategy and data extraction policy. HdB established the statistical approach contained within this protocol. All authors also drafted and edited the manuscript and approved the final version prior to submission.

All authors act as guarantors of this protocol.

\section{References}

1. Meara JG, Leather AJM, Hagander L, Alkire BC, Alonso N, Ameh EA, et al. Global Surgery 2030: evidence and solutions for achieving health, welfare, and economic development. Lancet. 2015 Aug;386(9993):569-624.

2. Shrime MG, Sleemi A, Ravilla TD. Charitable Platforms in Global Surgery: A Systematic Review of their Effectiveness, Cost-Effectiveness, Sustainability, and Role Training. World J Surg. 2015 Jan 29;39(1):10-20.

3. Bradley D, Honeyman C, Patel V, Zeolla J, Lester L, Eshete M, et al. Smartphones can be used for patient follow-up after a surgical mission treating complex head and neck disfigurement in Ethiopia: Results from a prospective pilot study. J Plast Reconstr Aesthetic Surg. 2020 Nov;In press.

4. Honeyman C, Patel V, Yonis E, Fell M, Demissie Y, Eshete M, et al. Long-term outcomes associated with short-term surgical missions treating complex head and neck disfigurement in Ethiopia: A retrospective cohort study. J Plast Reconstr Aesthetic Surg. 2020 May;73(5):951-8.

5. Shah SC, Kayamba V, Peek RM, Heimburger D. Cancer Control in Low- and Middle-Income Countries: Is It Time to Consider Screening? J Glob Oncol. 2019 Dec;(5):1-8.

6. Semer NB, Sullivan SR, Meara JG. Plastic surgery and global health: How plastic surgery impacts the global burden of surgical disease. J Plast Reconstr Aesthetic Surg. 2010 Aug;63(8):1244-8.

7. The World Health Organisation. The World Health Organisation. Injuries and Violence: The Facts [Internet]. 2010. Available from: http://scholar.google.com/scholar? 
hl=en\&btnG=Search\&q=intitle:Injuries+and+Violence:+The+Facts\#0 Date Accessed 22 November 2020

8. Hazari A, Cole R, Fowler C, Schache A, Nugent M, Ho M, et al. The British Association of Plastic Reconstructive and Aesthetic Surgeons First UK National Flap Registry Report. 2019.

9. Carney MJ, Weissler JM, Tecce MG, Mirzabeigi MN, Wes AM, Koltz PF, et al. 5000 Free Flaps and Counting. Plast Reconstr Surg. 2018 Apr;141(4):855-63.

10. Lewin J, Puri A, Quek R, Ngan R, Alcasabas AP, Wood D, et al. Management of sarcoma in the AsiaPacific region: resource-stratified guidelines. Lancet Oncol. 2013 Nov;14(12):e562-70.

11. Ducic I, Rao S, Attinger C. Outcomes of Microvascular Reconstruction of Single-Vessel Lower Extremities: Limb Salvage versus Amputation. J Reconstr Microsurg. 2009 Nov 10;25(08):475-8.

12. Millard DR, Dembrow V, Shocket E, Zavertnik J, Clinton-Thomas C. Immediate reconstruction of the resected mandibular arch. Am J Surg. 1967 Oct;114(4):605-13.

13. Habib AMA, Hassan SA. The feasibility of rib grafts in long span mandibular defects reconstruction: A long term follow up. J Cranio-Maxillofacial Surg [Internet]. 2019;47(1):15-22. Available from: https://doi.org/10.1016/j.jcms.2018.11.002

14. Lou C, Yang X, Hu L, Hu Y, S.P. Loh J, Ji T, et al. Oromandibular reconstruction using microvascularized bone flap: report of 1038 cases from a single institution. Int J Oral Maxillofac Surg. 2019;48(8):1001-8.

15. Nangole WF, Khainga S, Aswani J, Kahoro L, Vilembwa A. Free Flaps in a Resource Constrained Environment: A Five-Year Experience-Outcomes and Lessons Learned. Plast Surg Int. 2015;2015(September):1-6.

16. Nakarmi KK, Rochlin DH, Basnet SJ, Shakya P, Karki B, Magar MG, et al. Review of the First 108 Free Flaps at Public Health Concern Trust-Nepal Hospitals: Challenges and Opportunities in Developing Countries. Ann Plast Surg. 2018;81(5):565-70.

17. Trivedi NP, Trivedi P, Trivedi $\mathrm{H}$, Trivedi S, Trivedi N. Microvascular free flap reconstruction for head and neck cancer in a resource-constrained environment in rural India. Indian J Plast Surg. 2013;

18. Schaverien M V., Hamilton SA, Fairburn N, Rao P, Quaba AA. Lower Limb Reconstruction Using the Islanded Posterior Tibial Artery Perforator Flap. Plast Reconstr Surg. 2010 Jun;125(6):1735-43.

19. Wong JKF, Deek N, Hsu C-C, Chen H-Y, Lin C-H, Lin C-H. Versatility and "flap efficiency" of pedicled perforator flaps in lower extremity reconstruction. J Plast Reconstr Aesthetic Surg. 2017 Jan;70(1):67-77.

20. Olawoye OA, Ademola SA, lyun K, Michael A, Oluwatosin 0 . The reverse sural artery flap for the reconstruction of distal third of the leg and foot. Int Wound J. 2014 Apr;11(2):210-4.

21. Bertrand B, Honeyman CS, Emparanza A, McGurk M, Ousmane Hamady IE, Schmidt A, et al. TwentyFive Years of Experience with the Submental Flap in Facial Reconstruction. Plast Reconstr Surg. 2019 Jun;143(6):1747-58. 
22. Banda $\mathrm{CH}$, Georgios $\mathrm{P}$, Narushima $\mathrm{M}$, Ishiura R, Fujita $\mathrm{M}$, Goran J. Challenges in global reconstructive microsurgery: The sub-Saharan african surgeons' perspective. JPRAS Open. 2019 Jun;20:19-26.

23. Huijing MA, Marck KW, Combes J, Mizen KD, Fourie L, Demisse Y, et al. Facial reconstruction in the developing world: a complicated matter. Br J Oral Maxillofac Surg. 2011 Jun;49(4):292-6.

24. Shamseer L, Moher D, Clarke M, Ghersi D, Liberati A, Petticrew M, et al. Preferred reporting items for systematic review and meta-analysis protocols (PRISMA-P) 2015: elaboration and explanation. BMJ. 2015 Jan 2;349(jan02 1):g7647-g7647.

25. Sideri S, Papageorgiou SN, Eliades T. Registration in the international prospective register of systematic reviews (PROSPERO) of systematic review protocols was associated with increased review quality. J Clin Epidemiol. 2018 Aug;100:103-10.

26. Higgins JPT, Thomas J, Chandler J, Cumpston M, Li T, Page MJ WV. Cochrane Handbook for Systematic Reviews of Interventions version 6.0 (updated July 2019). Cochrane, 2019. Handbook. 2019;

27. Moher D, Liberati A, Tetzlaff J, Altman DG. Preferred reporting items for systematic reviews and meta-analyses: the PRISMA statement. BMJ. 2009 Jul 21;339(jul21 1):b2535-b2535.

28. The World Bank. World Bank Country and Lending Groups [Internet]. [cited 2021 Jan 6]. Available from: https://datahelpdesk.worldbank.org/knowledgebase/articles/906519-world-bank-country-andlending-groups Date Accessed 6 January 2021

29. Richardson WS, Wilson MC, Nishikawa J, Hayward RS. The well-built clinical question: a key to evidence-based decisions. Vol. 123, ACP journal club. 1995. p. A12-13.

30. Shanker MK, Rajan A, Hemant B, Kumar DA. Outcome of 1000 free flap head and neck reconstructions at a tertiary cancer care institute in India. Eur J Plast Surg. 2020 Jun 3;

31. Fischer JP, Nelson JA, Sieber B, Cleveland E, Kovach SJ, Wu LC, et al. Free Tissue Transfer in the Obese Patient. Plast Reconstr Surg. 2013 May;131(5):681e-692e.

32. Perisanidis C, Herberger B, Papadogeorgakis N, Seemann R, Eder-Czembirek C, Tamandl D, et al. Complications after free flap surgery: Do we need a standardized classification of surgical complications? Br J Oral Maxillofac Surg. 2012;50(2):113-8.

33. Sterne JAC, Savović J, Page MJ, Elbers RG, Blencowe NS, Boutron I, et al. RoB 2: a revised tool for assessing risk of bias in randomised trials. BMJ. 2019 Aug 28;14898.

34. Sterne JA, Hernán MA, Reeves BC, Savović J, Berkman ND, Viswanathan M, et al. ROBINS-l: a tool for assessing risk of bias in non-randomised studies of interventions. BMJ. 2016 Oct 12;i4919.

35. Group OL of EW. The Oxford Levels of Evidence 2 [Internet]. 2011 [cited 2019 Sep 16]. Available from: https://www.cebm.net/2016/05/ocebm-levels-of-evidence/ Date Accessed 22 November 2020

36. DerSimonian R, Laird N. Meta-analysis in clinical trials. Control Clin Trials. 1986 Sep;7(3):177-88.

37. Guyatt GH, Oxman AD, Vist GE, Kunz R, Falck-Ytter Y, Alonso-Coello P, et al. GRADE: an emerging consensus on rating quality of evidence and strength of recommendations. BMJ. $2008 \mathrm{Apr}$ 26;336(7650):924-6. 
38. Hendriks TCC, Botman M, Rahmee CNS, Ket JCF, Mullender MG, Gerretsen B, et al. Impact of shortterm reconstructive surgical missions: a systematic review. BMJ Glob Heal. 2019 Apr;4(2):e001176.

39. Matošević M, Pegan A, Sambunjak D, Solter D, Vagić D, Rašić I, et al. Significance of Anthropometric and Nutritive Factors in Oral and Oropharyngeal Cancer Patients Undergoing Free Flap Reconstruction. J Oral Maxillofac Surg. 2020 Apr;78(4):644-50.

\section{Supplementary Files}

This is a list of supplementary files associated with this preprint. Click to download.

- PRISMAPChecklist.docx 